\title{
Evolución de la potencia absoluta, relativa e índices de ritmos electroencefalográficos en estudiantes de primaria, licenciatura y maestría
}

\author{
Héctor Brust-Carmona, ${ }^{1}$ Marlene Galicia-Alvarado, ${ }^{1}$ Javier Alfaro Belmont, ${ }^{2}$ Ana Sánchez Quezada, ${ }^{3}$ \\ Jesica Cantillo-Negrete, ${ }^{4}$ Oscar Yáñez Suarez ${ }^{5}$
}

Artículo original

\section{ABSTRACT}

\section{Antecedents}

Cerebral function results from the electrical activity in glial-neuronal networks, integrated proactively through sensory, motor, and regulating interactions. These networks oscillate since early life and are modulated by diverse maturation factors, including educational processes.

\section{Objective}

To identify the power spectrum separated in delta $(z)$, theta $(\theta)$, alpha $1(\alpha 1)$, alpha $2(\alpha 2)$, beta $1(\beta 1)$, beta $2(\beta 2)$, and their topography in cerebral hemispheres of children, youngsters, and adults to establish $\mathrm{qEEG}$ indicators.

\section{Method}

We studied three groups of 16 participants each: elementary school children (CG), undergraduate students (UG), and graduate students (GG). Parents and participants granted their consent. The EEG was recorded (Nicolet) following the 10/20 system. Bipolar samples were analyzed. Absolute power (AP) was obtained with Fourier transform; its average (AAP) relative power (RP), and slow/fast frequencies and indices were calculated. Differences were assessed with Kruskal Wallis and Dunnet's comparison for subgroups.

\section{Results}

The AAP of six frequencies was higher in CG than in UG and GG. Frequencies were similar with exceptions correlating with topographic distribution. The $\delta / \alpha$ index was higher in CG with a particular topographic distribution, $\theta / \alpha$ varied more. RP of $\alpha$ was higher in $U G$ and GG than in CG; that of $\theta$ and $z$ were higher in some leads of CG.

\section{Discussion and conclusion}

During cerebral maturation, AP diminishes due to integration of more glial-neuronal ensembles, presenting greater asymmetry in a giving frequency. These profiles establish indicators for comparison with future EEG recordings.

Key words: qEEG power spectrum, qEEG indicators, alpha and beta 1 , delta and theta index over alpha.

\section{RESUMEN}

\section{Antecedentes}

La función cerebral resulta de la actividad eléctrica en redes glío-neuronales, integradas de forma activa por interacciones sensoriales, motoras y reguladores. Redes que oscilan desde la infancia y se modulan por diversos factores de maduración, incluyendo procesos educativos.

\section{Objetivo}

Identificar el espectro de potencia separado en delta $(z)$, theta $(\theta)$, alfa $1(\alpha 1)$, alfa $2(\alpha 2)$, beta $1(\beta 1)$ y beta $2(\beta 2)$ y su topografía en los hemisferios cerebrales de niños, jóvenes y adultos para establecer indicadores EEGc.

\section{Método}

Se estudiaron tres grupos de 16 participantes: niños en primaria $(G N)$, estudiantes de licenciatura (GL) y de maestría (GM). Los padres y los participantes otorgaron su consentimiento. En el aparato Nicolet se registró el EEG utilizando el sistema 10/20. Se analizaron muestras bipolares, con T Fourier se obtuvo la potencia absoluta (PA), se calculó su promedio (PPA), la potencia relativa (PR) y los índices de frecuencias lentas/rápidas. Las diferencias se evaluaron con Kruskal Wallis y la comparación de Dunnet para sub-grupos.

\section{Resultados}

El PPA de seis frecuencias fue mayor en GN que en $G L$ y GM. En éstos fue similar con excepciones, relacionadas con la distribución topográfica. El índice $\delta / \alpha$ fue mayor en $\mathrm{GN}$ con particular distribución topográfica y $\theta / \alpha$ fue más variable. La PR de $\alpha$ fue mayor en $\mathrm{GL}$ y GM comparados con $G N$, la de $\theta$ y de $z$ en algunas derivaciones, fueron mayores en el $\mathrm{GN}$.

\section{Discusión y conclusión}

En la maduración cerebral disminuye la PA debido a la integración de más ensambles glío-neuronales, que presentan mayor asimetría en determinada frecuencia. Estos perfiles establecen indicadores para compararlos con futuros registros EEG.

Palabras clave: Espectro de potencia EEGc, indicadores EEGc, alfa y beta 1 y 2 , índice delta y theta sobre alfa.

Laboratorio EEG. Dirección de Investigación. Instituto Nacional de Rehabilitación (INR).

Medicina Conductual. Facultad de Psicología, Universidad Nacional Autónoma de México (UNAM).

Servicio de Electrodiagnóstico, INR.

Subdirección de Investigación Tecnológica, INR.

Laboratorio de Neuroimagenes, Universidad Autónoma Metropolitana Iztapalapa (UAMI).

Correspondencia: Dr. Héctor Brust-Carmona. Laboratorio de Electroencefalografía, Dirección de Investigación. Instituto Nacional de Rehabilitación. Calz. México- Xochimilco 289, Arenal Guadalupe. Tlalpan, 14389, México DF. E-mail: hbrust@inr.gob.mx brusthector@gmail.com

Recibido primera versión: 6 de noviembre de 2014. Segunda versión: 24 de septiembre de 2015. Aceptado: 1 de diciembre de 2015. 


\section{INTRODUCCIÓN}

El análisis del espectro de potencia en diferentes frecuencias clasificadas como ritmos del EEG cuantitativo (EEGc) ha adquirido un nuevo impulso por tres vertientes: 1) Identificar y definir la participación de dichas frecuencias en redes glío-neuronales que se activan en vigilia y se modulan por la estimulación sensorial interna y externa, ${ }^{1-4}$ 2) La sintonización de circuitos cerebrales senso-motores y de asociación en una frecuencia específica. Lo cual, se compara con engramas previos resultando las sensaciones-percepciones e integración de respuestas sensitivo-motoras. ${ }^{5}$ Dicha sincronización puede representar funciones cognitivas subconscientes y conscientes ${ }^{6-7}$ y 3) Establecer marcadores EEGc que se utilicen para identificar alteraciones funcionales con repercusión en la conducta. ${ }^{8}$

Diferentes investigaciones del EEGc han mostrado relación entre la maduración cerebral con disminución de la potencia de frecuencias lentas con aumento de las rápidas, principalmente de alfa, beta y de gama. ${ }^{9}$

Sin embargo, es posible que el desarrollo cerebral resulte: 1) De una disminución o aumento selectivo de neuronas tanto corticales como subcorticales, 2) De las sinapsis entre axones y dendritas activadas por neurotransmisores y neuromoduladores, así como de sinapsis eléctricas, integrando redes glío-neuronales que se sintonizan en una frecuencia y que participan en manifestaciones conductuales, ${ }^{10-12}$ las cuales se adquieren a lo largo de la vida.

La ontogénesis de dichas redes se inicia en la gestación y continúa desde el nacimiento, agregándose las interacciones con el ambiente interno y externo, especialmente en los periodos críticos del desarrollo. ${ }^{13}$ De tal manera, que al aumentar la funcionalidad de redes cerebrales se manifiestan las conductas reflejas pre-programadas, las cuales se modifican de acuerdo a los resultados de la interacción con los cambios del ambiente, adquiriendo los procesos cognitivos responsables de la homeostasis, ${ }^{14}$ incluso se ha propuesto que se integran procesos alostásicos (circuitos que previenen cambios extremos de la homeostasis), que aseguran la supervivencia individual y grupal, aceptando al cerebro como una organización proactiva y no únicamente respondiente. ${ }^{15}$

Se han relacionado las frecuencias rápidas con procesos cognitivos y las lentas con alteraciones cerebrales. ${ }^{16}$ Sin embargo, se ha descrito que las frecuencias lentas como delta $\mathrm{y}$ theta participan en la regulación de funciones vegetativas y emocionales, ${ }^{17,18}$ pero sobretodo, el ritmo theta participa en los procesos de la memoria, ${ }^{12}$ ya sea por sí solo o interactuando con alfa y beta, o incluso con gama. ${ }^{15}$

Por lo anterior, proponemos que la coherencia de redes neuronales en una frecuencia genera procesos conductuales internos y externos, incluyendo lo cognitivo. Estas redes aumentan tanto en relación con la edad, como con la participación en los procesos de aprendizaje. ${ }^{19}$
Dichos procesos dinámico-funcionales se incrementan conforme aumentan las estimulaciones sensoriales, se regulan las respuestas y se organizan electromorfogramas de diferentes frecuencias en múltiples circuitos en serie y en paralelo. Una vez integrados, esta información se almacena (memoria), estableciendo la condición funcional basal que proponemos como bloques funcionales de la conducta.

Como resultado de las respuestas se generan nuevas señales tanto en modificaciones de las oscilaciones (desincronización/ sincronización) como trenes de potenciales de acción, señales que se comparan y relacionan con datos que identifican la variación de la homeostasis y así, sucesivamente, se integran redes dinámicas cerebrales que participan en diversas funciones que resultan de diferentes índices de correlación entre frecuencias y redes de diversas áreas cerebrales. ${ }^{20}$

Por lo tanto, en individuos en reposo y con la mayor tranquilidad posible, los circuitos neuronales manifiestan aquellas frecuencias que han establecido como electromorfogramas basales ${ }^{21}$ y su potencia absoluta será menor mientras más se integren ensambles de circuitos dentro de un rango de frecuencia, condición que es esencial para futuras interacciones con el ambiente. Dentro de esta integración predomina la organización de circuitos que funcionan en el rango de alfa, ${ }^{22}$ frecuencias que son modificadas por potenciales postsinápticos facilitadores e inhibidores y que controlan los trenes de potenciales de acción. ${ }^{23}$ Estos mecanismos integran la actividad en ensambles glío-neuronales en cada momento, reconstruyendo redes neuronales funcionales, ${ }^{24}$ que incluyen interneuronas facilitadoras e inhibidoras..$^{25}$

Los procesos de "maduración" del ciclo vigilia-sueño, de la atención y del aprendizaje resultan de la desincronización (activación) o de la sincronización (inhibición) de frecuencias específicas en determinados circuitos neuronales, relacionados con eventos específicos. Lopes da Silva propone que la activación representa el proceso de permitir una determinada función, mientras que la inhibición corresponde a impedirla. ${ }^{26}$

Para conocer dichos procesos, a lo largo de diferentes etapas del desarrollo humano, es necesario caracterizar la potencia absoluta (PA) del espectro de las oscilaciones del EEG con su topografía en sujetos en reposo con los ojos cerrados. Con los resultados, esperamos extraer los indicadores EEGc que identifiquen adaptaciones funcionales las cuales representan la maduración cerebral. Estos indicadores, en una primera etapa, serán comparados con nuevos registros y, en una segunda, para aumentar el tamaño de la muestra y analizarla con tecnologías de computación especializadas, incluyendo las evaluaciones estadísticas de precisión y confiabilidad. Esperamos poder proponerlos como normativos para apoyar procesos diagnósticos y establecer la base cuantitativa para evaluar la eficiencia de intervenciones de la rehabilitación integral en los padecimientos cerebrales; en particular, en alteraciones del aprendizaje y en enfermedades cerebrales adquiridas. 
Por lo tanto, en este trabajo presentamos resultados de niños en enseñanza primaria, estudiantes de licenciatura y maestría para establecer similitudes y diferencias que permitan identificar, en los dominios temporales y de potencia, las modificaciones significativas e inferir la integración de redes neuronales que se manifiesten en una determinada frecuencia y que reflejen posibles indicadores funcionales.

\section{MÉTODO}

Descriptivo, transversal de 16 niños. Los GN de siete a 10 años (8.5 \pm 1.6 años), en total nueve niños de escuelas primarias públicas que asisten al INR por diferentes razones no clínicas y cuyo examen psicológico (NEUROPSI Atención y Memoria; Ostrosky-Solís et al., 2003) y neurológico (historia clínica del neurólogo del servicio de Neurorehabilitación) no detectaron anormalidad (criterios de inclusión). Los GL (de $21.5 \pm 1.32$ años de edad) en total 16 estudiantes, todas mujeres seleccionadas al azar de una base de datos de 68 estudiantes de licenciatura en el INR que habían sido estudiadas anteriormente. Los GM (de $26.38 \pm 2.25$ años de edad) fueron 16 participantes (ocho hombres) seleccionados de entre 30 estudiantes de maestría en Ingeniería Eléctrica, a los cuales se les registró su EEG para analizar cambios del ritmo alfa en la región motora al efectuar un determinado movimiento o cuando se lo imaginaron.

Tanto los padres o tutores, como los participantes adultos debidamente informados, procedieron a firmar el formato de aprobación del INR. Los protocolos correspondientes fueron previamente aprobados por los Comités de Investigación y de Ética del INR.

A los participantes de los dos primeros grupos se les indicó que estarían acostados en decúbito dorsal, la mayor parte del tiempo con los ojos cerrados (OC) y, en ciertos momentos, se les pediría que lentamente levantaran el párpado superior, sin mover los globos oculares y fijaran su vista en un punto del techo, lo que se ensayó varias veces antes del registro (mayor número de ensayos en los escolares). Los del tercer grupo estuvieron en un sillón reclinable con los ojos cerrados; también se les indicó que abrieran los ojos y enfocaran un punto determinado.

Para todos los registros se utilizó un EEG digital (Viasys Nicolet One VIASYC Health, Inc., USA), colocando los electrodos de acuerdo con el procedimiento 10/20, poniendo especial énfasis en la distancia inter-electrodos y en mantener la impedancia entre $5-10 \mathrm{k} \Omega$ durante todo el estudio. Además, se registraron movimientos palpebrales y de los ojos, colocando electrodos en los vértices de las aperturas palpebrales, y el EKG, colocando un electrodo en el hemitórax izquierdo. Los análisis se efectuaron en las conexiones bipolares de las derivaciones parasagitales (DPS) y laterales (DLA) en ambos hemisferios (AH). El EEG se acondicionó con filtro pasa-banda de $1.6 \mathrm{~Hz}$ a $40 \mathrm{~Hz}$ y uno de rechaza-banda de $60 \mathrm{~Hz}$. Del registro, se seleccionaron en el intervalo de OC tres ventanas de 12s, libres de artefactos. Para el análisis se utilizó la transformada de Fourier, obteniendo la potencia absoluta (PA) de la frecuencia $z$ (1.6 a 4.0 $\mathrm{Hz}), \theta(4.5 \mathrm{a} 8.0 \mathrm{~Hz}), \alpha 1$ (8.5 a 10), $\alpha 2$ (10.5 a $13 \mathrm{~Hz}), \beta 1$ (13.5 a $20 \mathrm{~Hz})$ y $\beta 2(20.5 \mathrm{a} 30 \mathrm{~Hz})$. Posteriormente, se calcularon los promedios de la PA (PPA), las potencias relativas (PR) y los índices $z / \alpha 1, \zeta / \alpha 2, \zeta / \beta 1, \gamma / \beta 2, \theta / \alpha 1$ o $\alpha 2, \theta / \beta 1$ o $\beta 2$, de

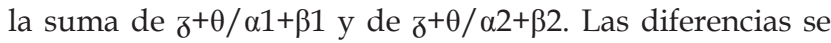
evaluaron con la prueba no paramétrica de Kruskal-Wallis y con la comparación de Dunnet para sub-grupos, con un intervalo de confianza $\alpha=0.05$.

\section{RESULTADOS Delta ( ()}

El PPA de z en las DLA y DPS de cada hemisferio, fue mayor en GN que en GL y GM; la diferencia fue estadísticamente significativa entre GN vs. GL y GM, pero no entre GL y GM, excepto en la derivación témporo-temporal de cada hemisferio, en la cual el PPA de z fue significativamente menor en GM que en GL. Los PPA fueron mayores en DPS en los tres grupos, pero la diferencia con las laterales no fue significativa (figura 1).

\section{Theta $(\theta)$}

El PPA de $\theta$ en las DLA fue significativamente mayor en GN que en el correspondiente del GL y GM, excepto en T3T5 de GL. Es notable el aumento del PPA en derivaciones témporo-temporales de los tres grupos y, en particular, en la témporo-occipital derecha de los niños, que fue el mayor valor de los tres grupos. En las DPS, el PPA de $\theta$ fue significativamente mayor en GN que en los otros dos grupos; aunque en todas las derivaciones fue menor en GM, no difiere significativamente del GL. Se observó mayor PPA en el HD de los tres grupos, en particular de los niños, en la derivación parieto-occipital derecha, pero la asimetría interhemisférica no fue significativa (figura 1 ).

\section{Alfa $1(\alpha 1)$}

El PPA de $\alpha 1$ de GN fue menor comparado con GL y GM, tanto en las DLA como en las DPS, significativamente para GM en todas las derivaciones y para GL sólo en fronto-frontales, témporo-occipitales y parieto-occipitales. El PPA fue similar en todas las derivaciones para los grupos GL y GM (figura 2).

\section{Alfa 2 (a2)}

La figura 2 muestra que el PPA disminuyó significativamente de GN a GM en F2F8 y entre GL vs. GM en T4T6. En las 

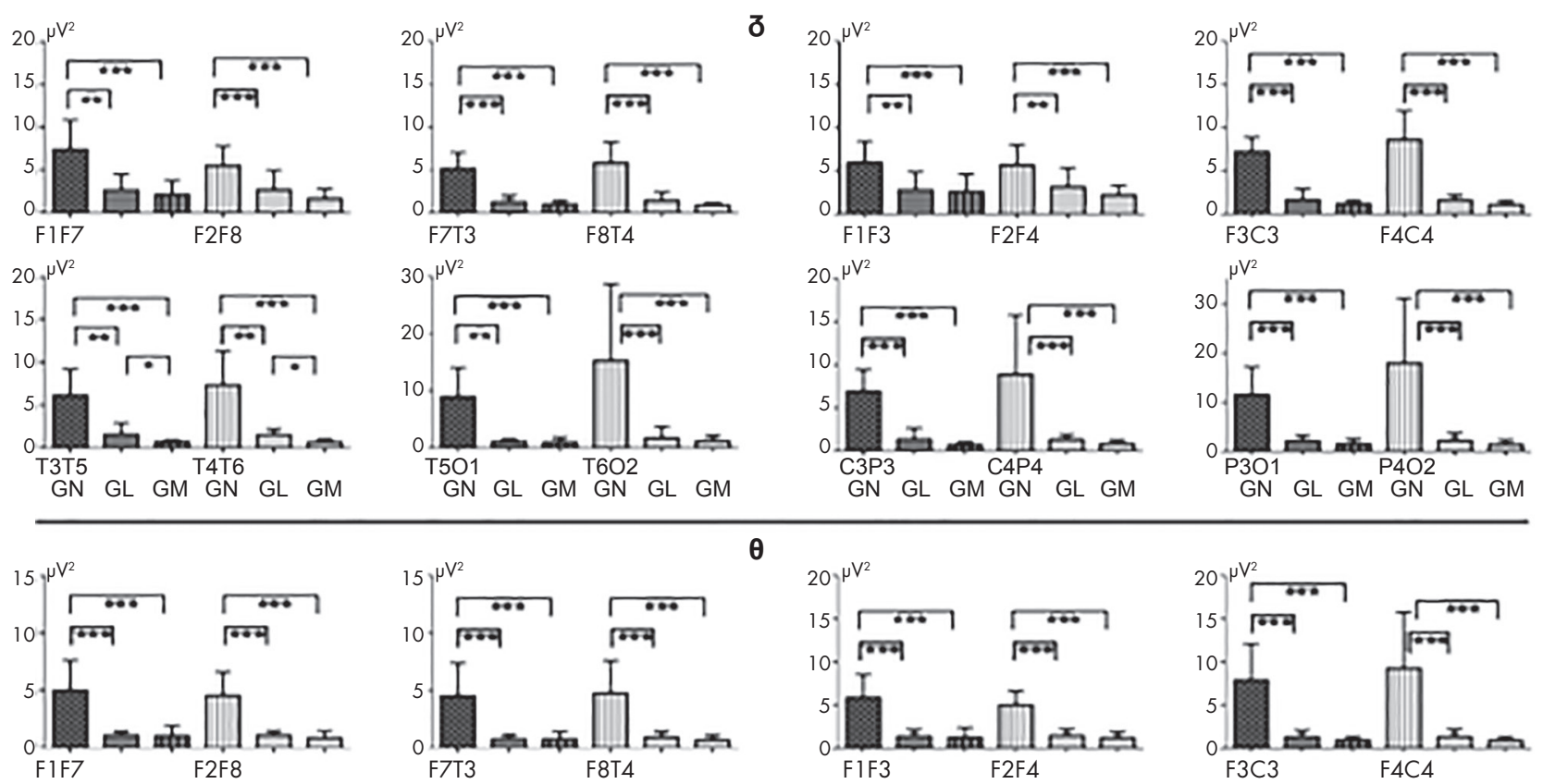

$\theta$
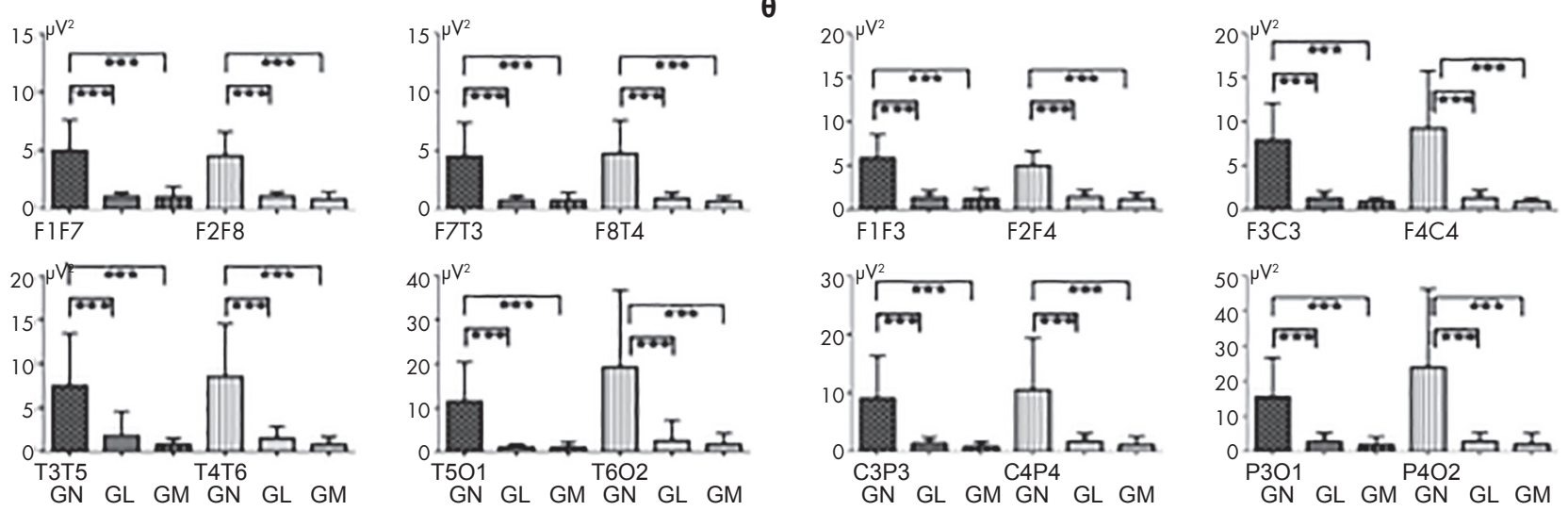

Figura 1. Comparación del PPA de ð (parte superior) y $\theta$ (parte inferior) en GN, GL y GM en las cuatro derivaciones laterales (lado izquierdo) y en las cuatro parasagitales (lado derecho). Las llaves marcan los grupos comparados, y los asteriscos $\left({ }^{*} p 0.05,{ }^{* *} p 0.001\right.$, $* * *$ p 0.0001 ) la significancia estadística.

DPS, disminuyó significativamente de GN vs. GL y GM en fronto-frontales, en todas las demás derivaciones los valores del PPA fueron similares.

\section{Beta $1(\beta 1)$}

El PPA de $\beta 1$ fue significativamente mayor en GN en todas las derivaciones. Además, se registró disminución del PPA de $\beta 1$ de GL a GM en las derivaciones témporo-temporales de $\mathrm{AH}$ y en las parieto-occipital derecha (figura 3 ).

\section{Beta 2 ( $\beta 2)$}

En la figura 3 se muestran los PPA de $\beta 2$ que difirieron entre los tres grupos. No se registraron diferencias significativas en las derivaciones fronto-frontales laterales y parasagitales ni en las centro-parietales izquierdas. Se registró disminución significativa de GN a GL en F2F8, F2F4 y F4C4 y entre GN y GM en todas las derivaciones, excepto en fronto-frontal lateral y parasagital izquierdas, en témporo-temporal izquierda y centro-parietal izquierda. Por último, los PPA de $\beta 2$ disminuyeron de GL a GM en derivaciones tém- poro-temporales, témporo-occipitales y parieto-occipitales de $\mathrm{AH}$.

\section{Comparación de los perfiles del PPA}

En GN, el perfil del PPA de $\precsim$ muestra un ascenso fronto-occipital, excepto en F7T3 que disminuye, presentando menor desviación estándar. Todos los valores mostraron gran variabilidad, siendo ésta mayor en GN que las correspondientes de los otros dos grupos. El perfil en GL y GM mostró descenso del PPA en la derivación fronto-temporal o en la fronto-central con posterior ascenso en las siguientes derivaciones y prácticamente fueron similares en ambos grupos.

El perfil de $\alpha 1$ fue de ascenso fronto-occipital en los tres grupos, con mayor intensidad y variabilidad en GN, a diferencia de los otros dos grupos, en los que su perfil es similar. El perfil del PPA de $\alpha 2$ fue también de ascenso en los tres grupos, con mayor intensidad y variabilidad en GN, seguido de GL y, al último, GM (figura 4).

El perfil de $\beta 1$ fue de disminución de fronto-frontal a fronto-temporal y fronto-central con posterior ascenso en las otras dos derivaciones en los tres grupos, pero con ma- 

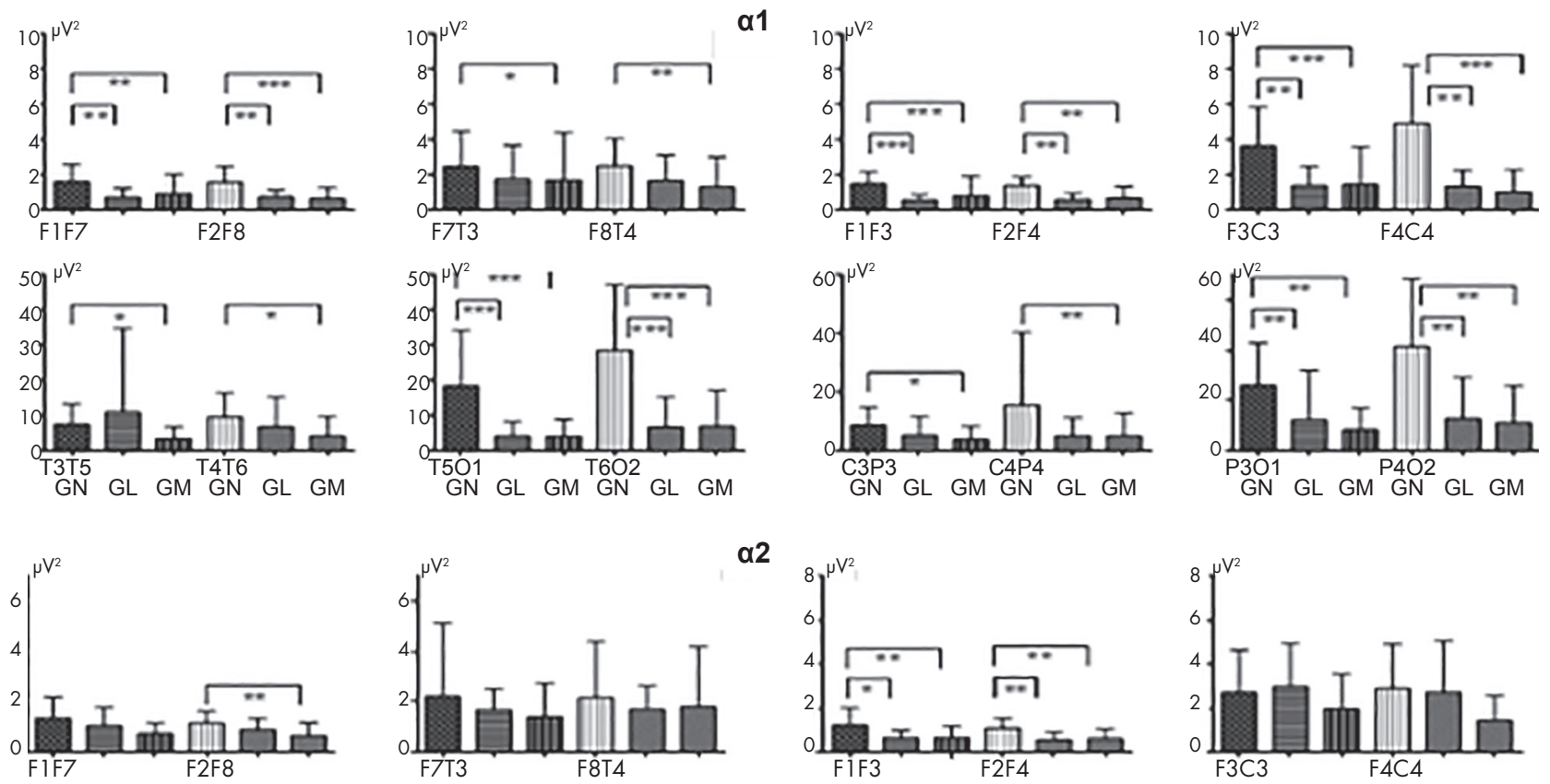

$\alpha 2$
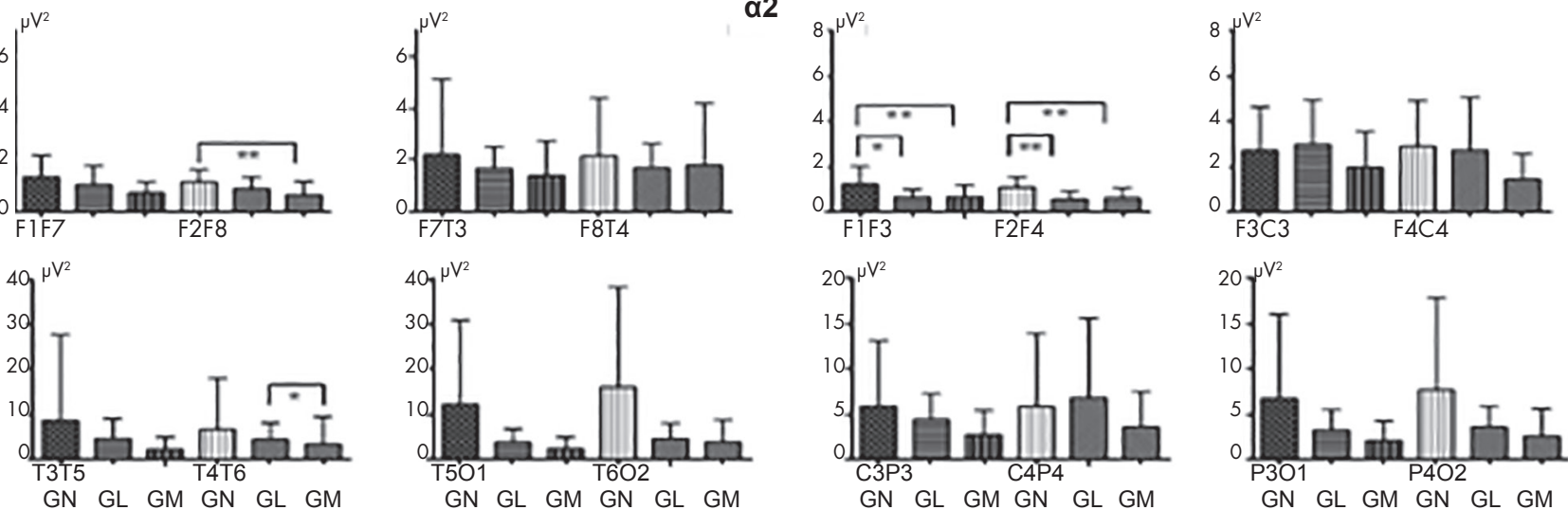

Figura 2. Comparación del PPA de $\alpha 1$ (parte superior) y $\alpha 2$ (parte inferior) en $G N, G L$ y $G M$, en las cuatro derivaciones laterales (lado izquierdo) y en las cuatro parasagitales (lado derecho). Las llaves marcan los grupos comparados, y los asteriscos ( ${ }^{*} \mathrm{p} 0.05,{ }^{*} \mathrm{p} 0.001$, $* * *$ p 0.0001 ) la significancia estadística.

yor PPA en GN, seguido de GL y con menor PPA y variabilidad en el GM. El perfil de $\beta 2$ en GN fue de disminución frontal en las dos siguientes derivaciones y de aumento en las posteriores. El GL presentó un perfil de ascenso en derivaciones témporo-temporales y témporo-occipitales de $\mathrm{AH}$, mientras que en DPS mostró descenso fronto-frontal a la siguiente derivación y aumento en las otras dos derivaciones. Por último, el perfil en GM es de descenso fronto-occipital en las DLA y DPS, presentando similar PPA.

\section{Comparación de la PR}

La PR de $z$ fue significativamente mayor en GN que en GL en todas las DLA y DPS, excepto en fronto-frontales y témporo-occipitales izquierdas; mientras que, comparado con GM, fue significativamente mayor en témporo-temporal, centro-parietal y parieto-occipital izquierdas, así como en todas las derivaciones derechas. Las PR fueron similares entre el GL y GM en esta frecuencia.

La PR de $\theta$ fue mayor en DLA izquierdas de niños y la diferencia fue significativa en las cuatro derivaciones. Las diferencias entre el GL y el GM fueron significativas en la témporo-temporal izquierda. La PR de $\theta$ del GN fue mayor en todas las DLA y las DPS del HD, excepto en fronto-frontal derecha. En GL y GM las diferencias fueron significativas en fronto-temporal y témporo-temporal del HD, mientras que en las parasagitales entre GL y GM no se detectaron diferencias significativas.

La PR de $\alpha 1$ en el HI fue mayor en GM, quedando en segundo lugar el GL, aunque entre estos dos las diferencias no fueron significativas y, en tercer lugar, quedó el GN.

La PR de $\alpha 2$ fue mayor en GL en las DLA de AH y la diferencia comparada con GN fue significativa, mientras que no lo fue en fronto-frontales de $\mathrm{AH}$. Por lo tanto, la menor PR se obtuvo en el GN, lo que se señala con un asterisco en el cuadro 1.

La PR de $\beta 1$ en DLA izquierdas fue mayor en GL y difirió en T5O1 del GN. En DPS izquierdas también fue mayor en GL con diferencia en F3C3 y P3O1 de GN. La mayor PR de $\beta 1$ en DLA correspondió a GM en F2F8 y F8T4, mientras que para el GL correspondió a T4T6 y T6O2 con diferencia significativa con respecto al GN en estas dos derivaciones. La mayor PR de $\beta 1$ en DPS la obtuvo el GL, excepto en F2F4, y difirió en F4C4 y P4O2 del GN. 

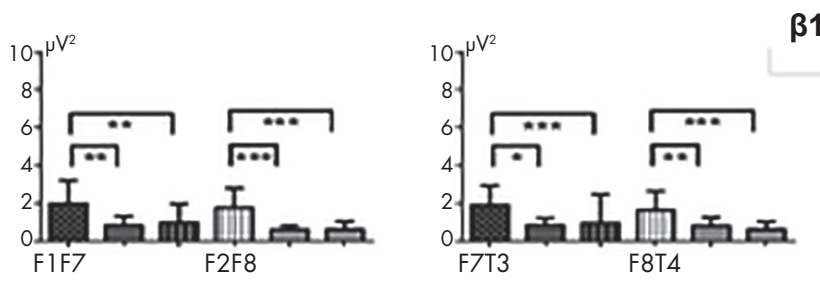

$\beta 1$
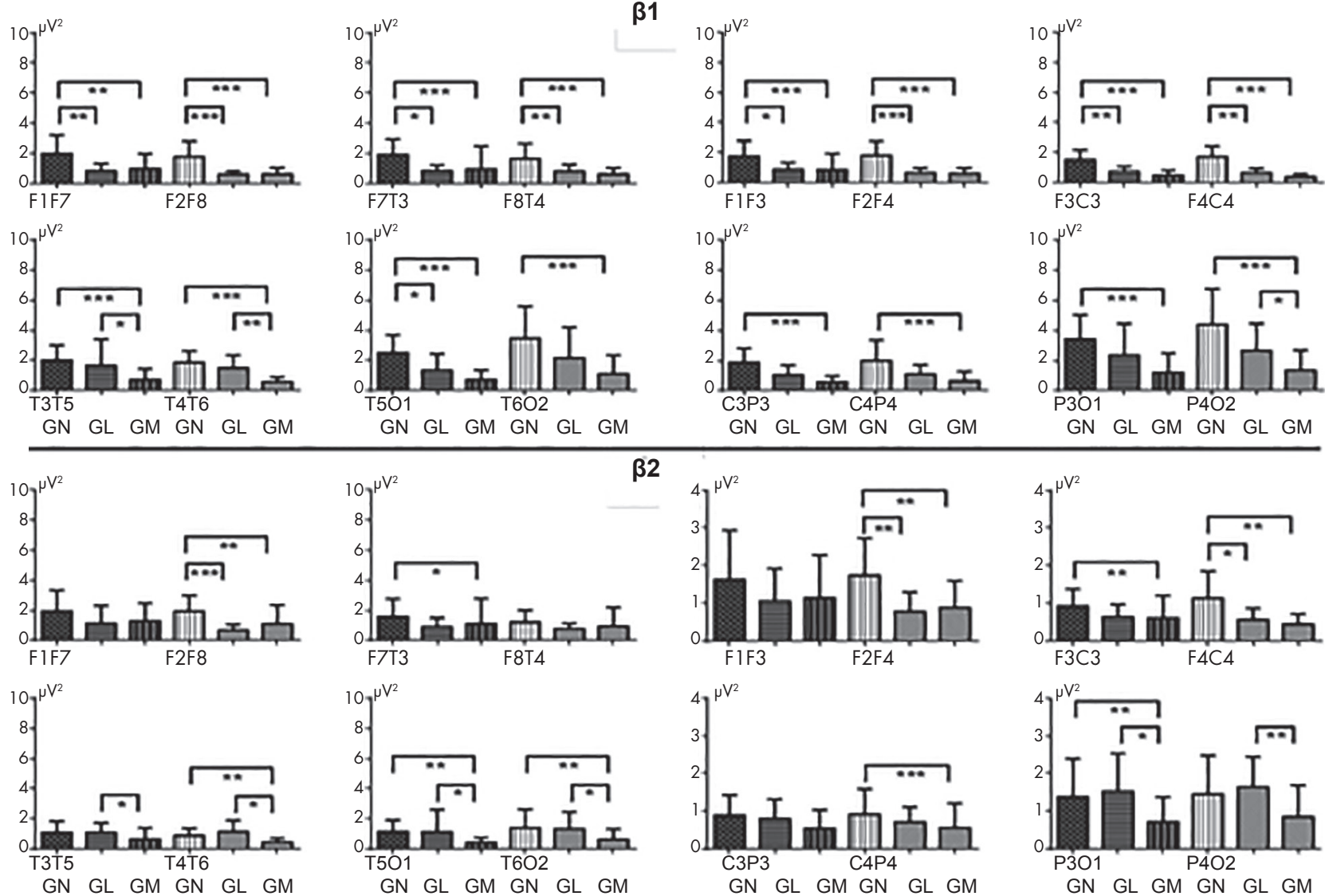

Figura 3. Comparación del PPA de $\beta 1$ (parte superior) y $\beta 2$ (parte inferior) en GN, GL y GM en las cuatro derivaciones laterales (lado izquierdo) y en las cuatro parasagitales (lado derecho). Las llaves marcan los grupos comparados, y los asteriscos ( ${ }^{*} \mathrm{p} 0.05,{ }^{*} \mathrm{p} 0.001$, $* * *$ p 0.0001 ) la significancia estadística.
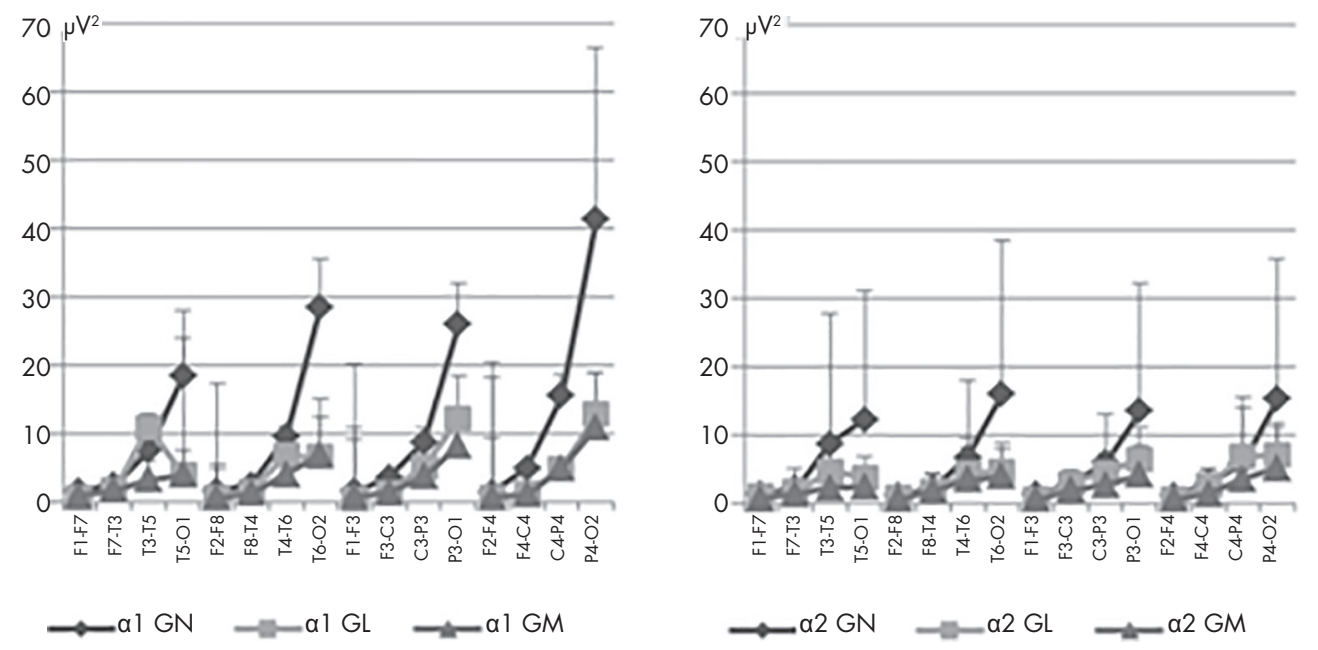

Figura 4. Perfil del PPA de $\alpha 1$ (parte derecha) y de $\alpha 2$ (parte izquierda) en relación con cada una de las derivaciones del GN GL y GM. Se muestra el perfil ascendente de mayor voltaje en los niños y un perfil muy similar en los otros dos grupos pero con menores intensidades y variabilidad, indicada por la desviación estándar. 


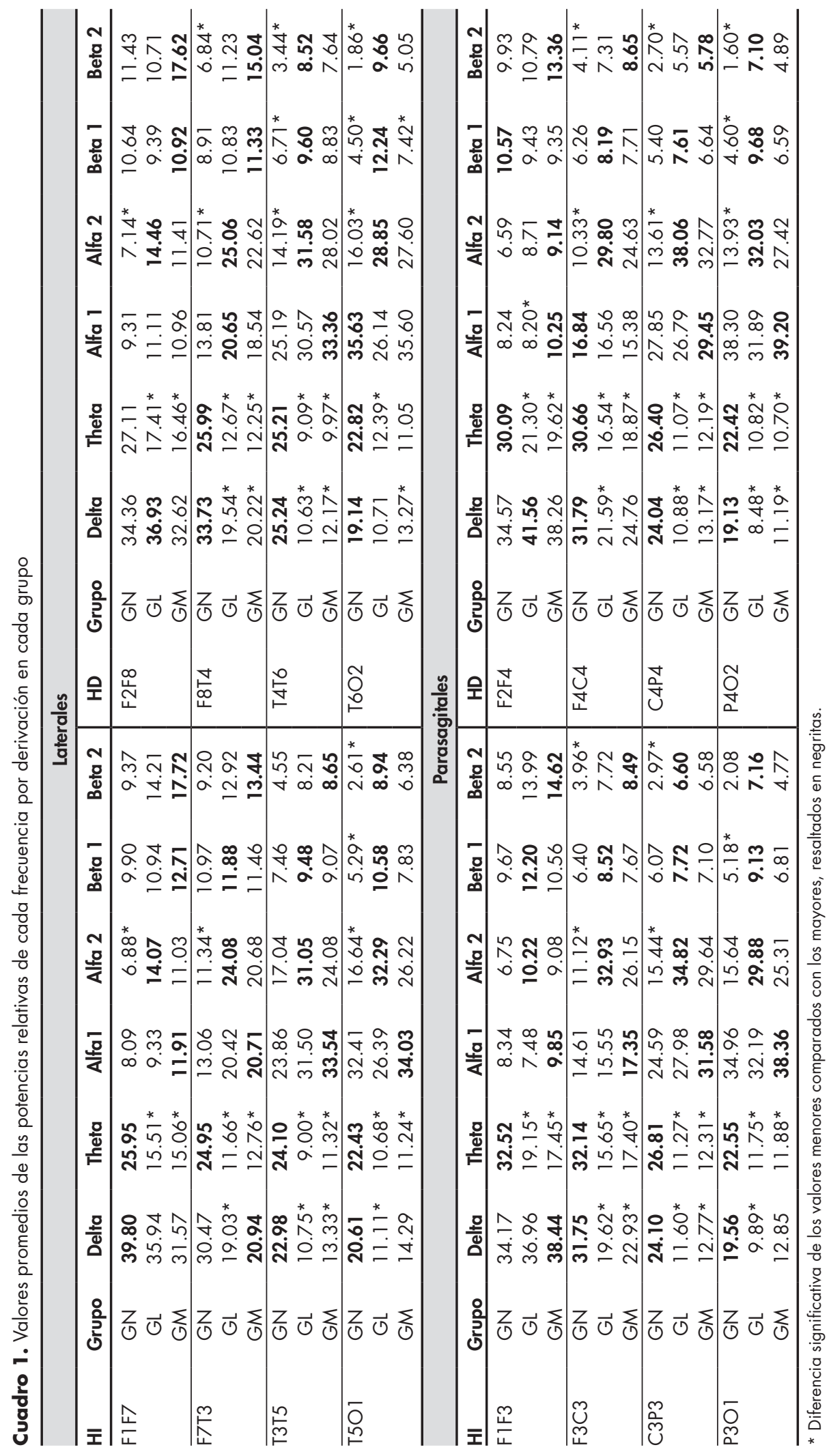




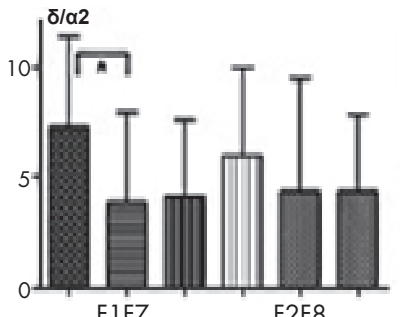

F1F7

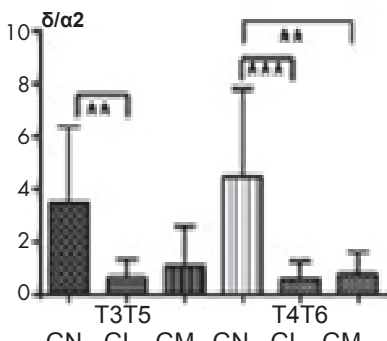

GN GL GM GN GL GM

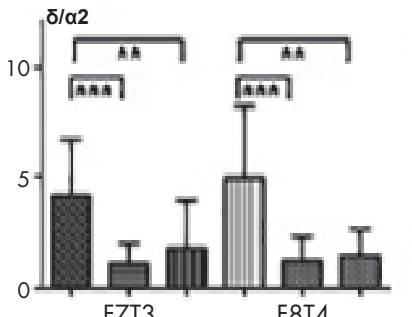

F7T3

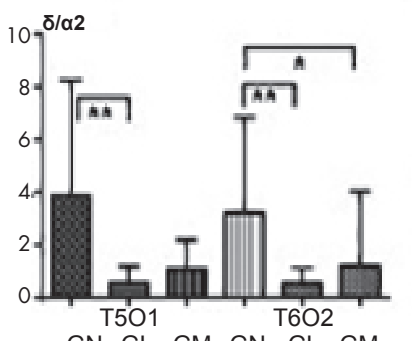

GN GL GM GN GL GM
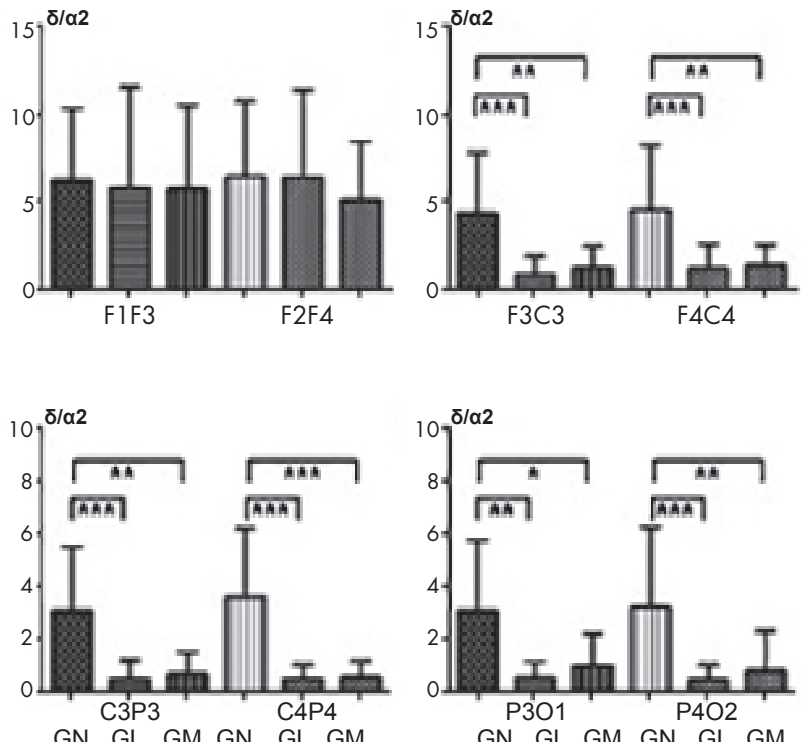

Figura 5. Las columnas representan los promedios y la línea ascendente la desviación estándar de los índices $\precsim / a 2$ en las diferentes derivaciones de cada grupo. Los asteriscos marcan el nivel de significancia entre las columnas indicadas.

La mayor PR de $\beta 2$, en DLA izquierdas la obtuvo GM en tres derivaciones anteriores y difirió en F1F7 del GN, mientras que la mayor PR la presentó el GL en T5O1 y difirió del GN en esta misma derivación. En DPS izquierdas, la mayor PR la obtuvo el GL, excepto en F1F3, y las tres primeras derivaciones difirieron del GN. La PR de $\beta 2$ en las dos primeras DLA derechas fue mayor en GM y difirió en F8T4 del GN, mientras que en las otras dos derivaciones fue mayor en GL y fue diferente del GN en ambas. En las dos derivaciones anteriores parasagitales derechas fue mayor en GM y diferente en F4C4 del GN, mientras que, en las dos posteriores, el mayor valor lo obtuvo GL y fue diferente del GN en ambas (cuadro 1).

El índice $z / \alpha 1$ en DLA fue mayor en GN que en GL y GM; el menor valor de éstos se registró en las DLA de AH, excepto en F2F8. En las DPS se registró un aumento de z/ $\alpha 1$ en fronto-frontales de $\mathrm{AH}$, aunque la diferencia sólo fue significativa en fronto-temporales de $\mathrm{AH}$.

El índice $z / \alpha 2$ disminuyó significativamente en todas las derivaciones del GL y GM comparado con GN (figura 5).

El índice $\theta / \alpha 1$ disminuye significativamente de GN a GL y GM en tres DLA, pero no en las témporo-occipitales de cada hemisferio. Mientras que en DPS sólo disminuyó de GN a GM, en las demás derivaciones se obtuvieron valores similares.

En contraste, el índice $\theta / \alpha 2$ disminuyó en GN respecto a los otros grupos, en todas las derivaciones, excepto en T5O1 para el GM (figura 6).

El índice $\theta / \beta 1$ disminuye en GN al compararla con los otros grupos en todas las derivaciones, excepto en F2F4. Los valores en GL y GM fueron muy similares (figura 7).
El índice $\theta / \beta 2$ disminuye de $\mathrm{GN}$ a los otros grupos, ex-

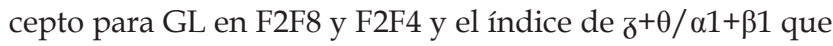
presentó valores similares en derivaciones témporo- $\mathrm{y}$ parieto-occipitales de $\mathrm{AH}$, así como en F1F3, F2F4 y F2F8. En las otras derivaciones, los valores fueron significativamente menores en GL y GM comparados con GN (figura 8).

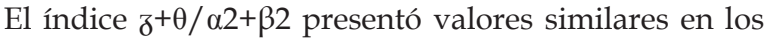
tres grupos en $\mathrm{F} 2 \mathrm{~F} 4$, y en todas las otras derivaciones los valores fueron menores para GL y GM.

\section{DISCUSIÓN Y CONCLUSIÓN}

La mayor PA de z en niños puede deberse a que los ensambles glío-neuronales generan flujos transmembrana iónicos lentos, produciendo campos eléctricos que coactivan otros grupos neuronales ${ }^{27}$ lo cual aumenta la PA registrada en el EEGc. Conforme se desarrollan y mielinizan las conexiones intracorticales se activan diversas sinapsis en diferentes neuronas. Ambos procesos integran ensambles glío-neuronales dinámicos que, de acuerdo a sus características intrínsecas, generan las oscilaciones de una frecuencia. Por lo tanto, se integran circuitos que se sintonizan en sub-frecuencias dentro de ese rango. ${ }^{28}$

Esta posible organización va separando funcionalmente los ensambles, aumentando los grupos que oscilan desfasados en una frecuencia $y$, por consiguiente, disminuye la PA de ésta.

La formación de circuitos también ocurre en el tallo cerebral, ${ }^{29}$ en los núcleos talámicos, ${ }^{30} \mathrm{y}$ en los ganglios basales, ${ }^{31}$ que al desarrollarse y mielinizarse las conexiones entre ellos establecen circuitos que funcionan dentro de 

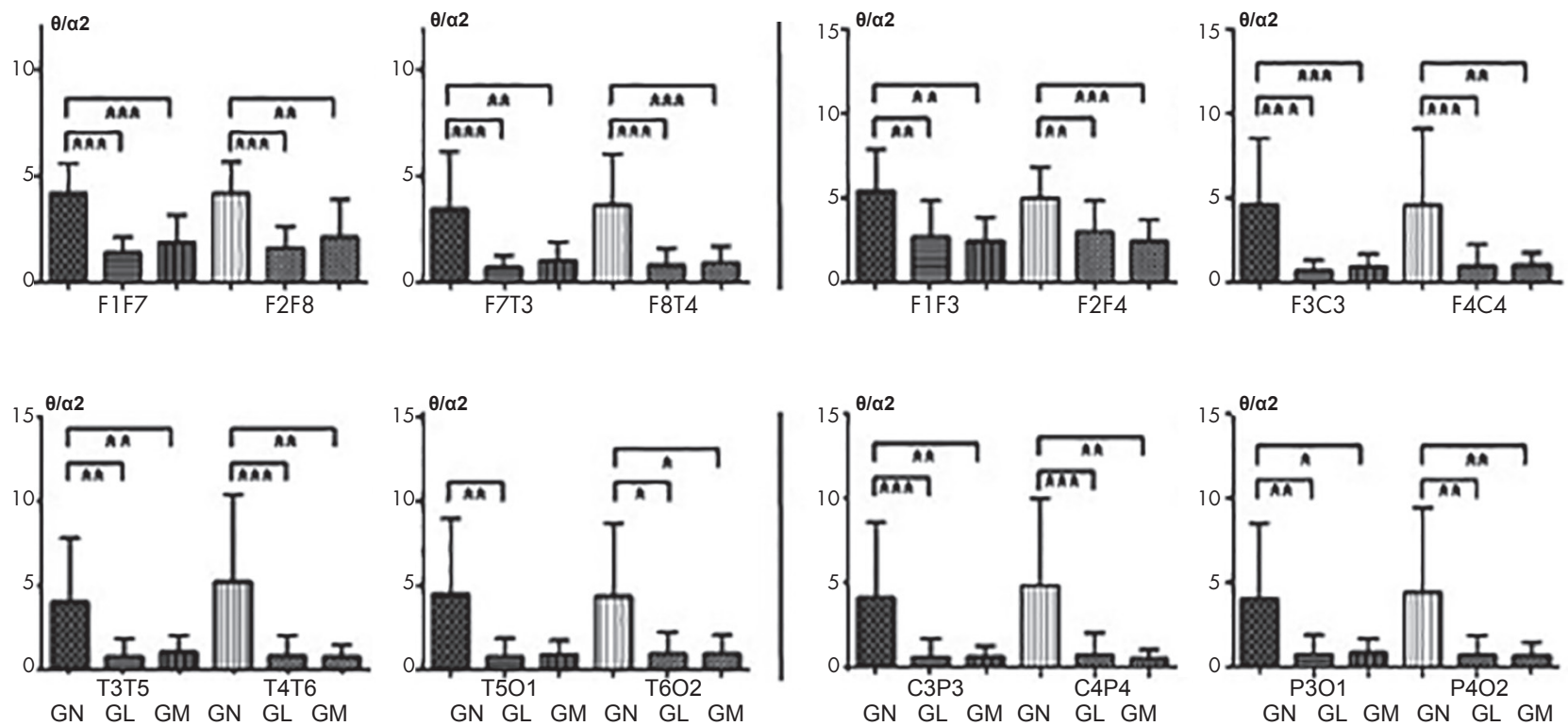

Figura 6. Las columnas representan los promedios, y la línea ascendente la desviación estándar de los índices $\theta / \alpha 2$ en las diferentes derivaciones de cada grupo. Los asteriscos señalan el nivel de significancia entre las columnas indicadas.
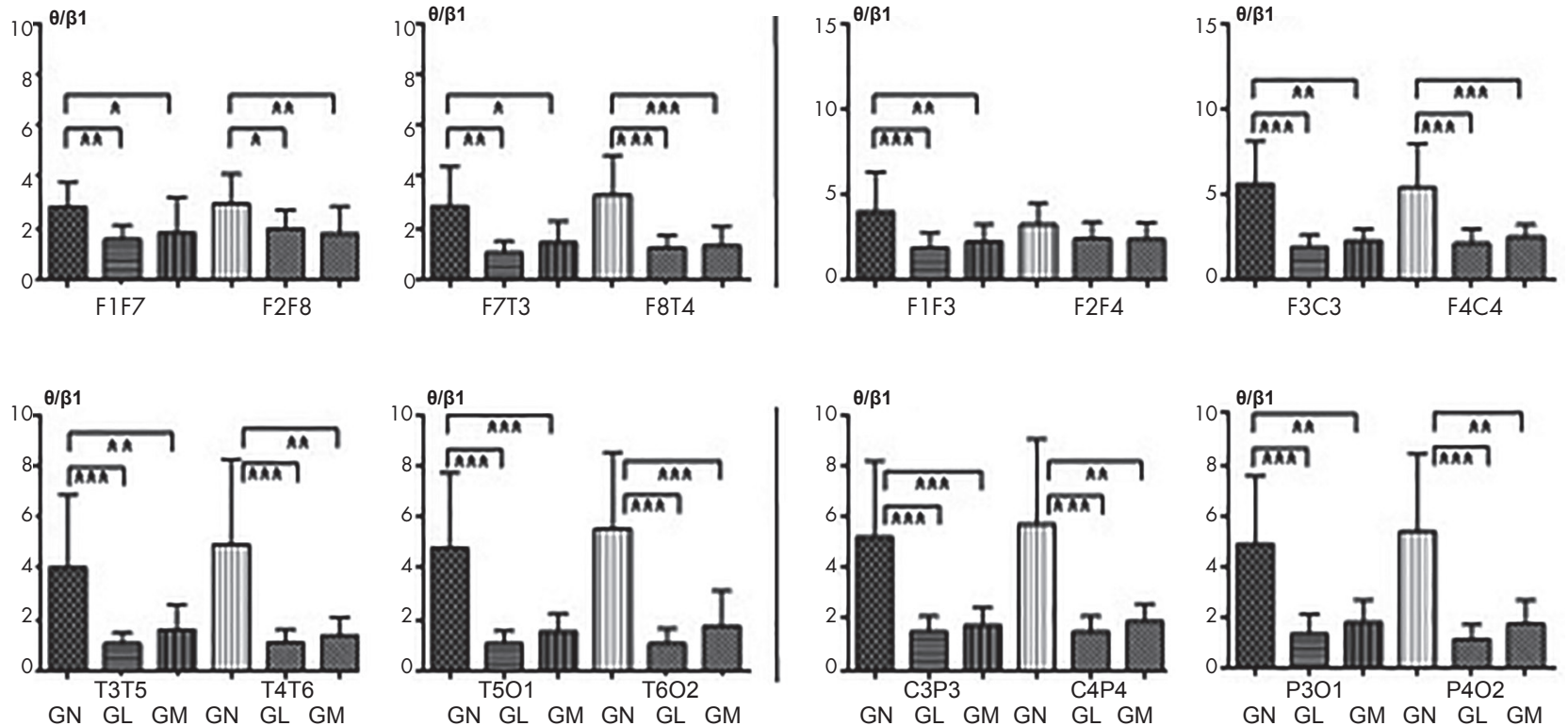

Figura 7. Las columnas representan los promedios, y la línea ascendente la desviación estándar de los índices $\theta / \beta 1$ en las diferentes derivaciones de cada grupo. Los asteriscos marcan el nivel de significancia entre las columnas indicadas.

esa frecuencia. Estos circuitos subcorticales se conectan con los corticales, formando redes más amplias que funcionan como sistemas activadores y de retro-activación con importante participación de interneuronas, principalmente inhibidoras, que sostienen las oscilaciones. ${ }^{32}$

Estas redes se sintonizan en una frecuencia que se relaciona con la conducta. Los escolares mostraron mayores PA en $z$ y $\theta$, mientras que en los estudiantes de licenciatura y de maestría fueron menores con similar magnitud entre éstos, lo que también se comprobó en la disminución de los índices de $z$ y $\theta$ sobre $\alpha$.

Existen resultados que apoyan la hipótesis alternativa de organización funcional en la maduración cerebral, que consiste en la integración de ensambles glío-neuronales que se sintonizan en una frecuencia de oscilaciones, ${ }^{33,34}$ por ejemplo, los que funcionan con ritmo $\alpha$ que se han relacionado con prácticamente todas las funciones corporales, y los ensambles que funcionan con ritmo $\beta$, que aparentemente se 

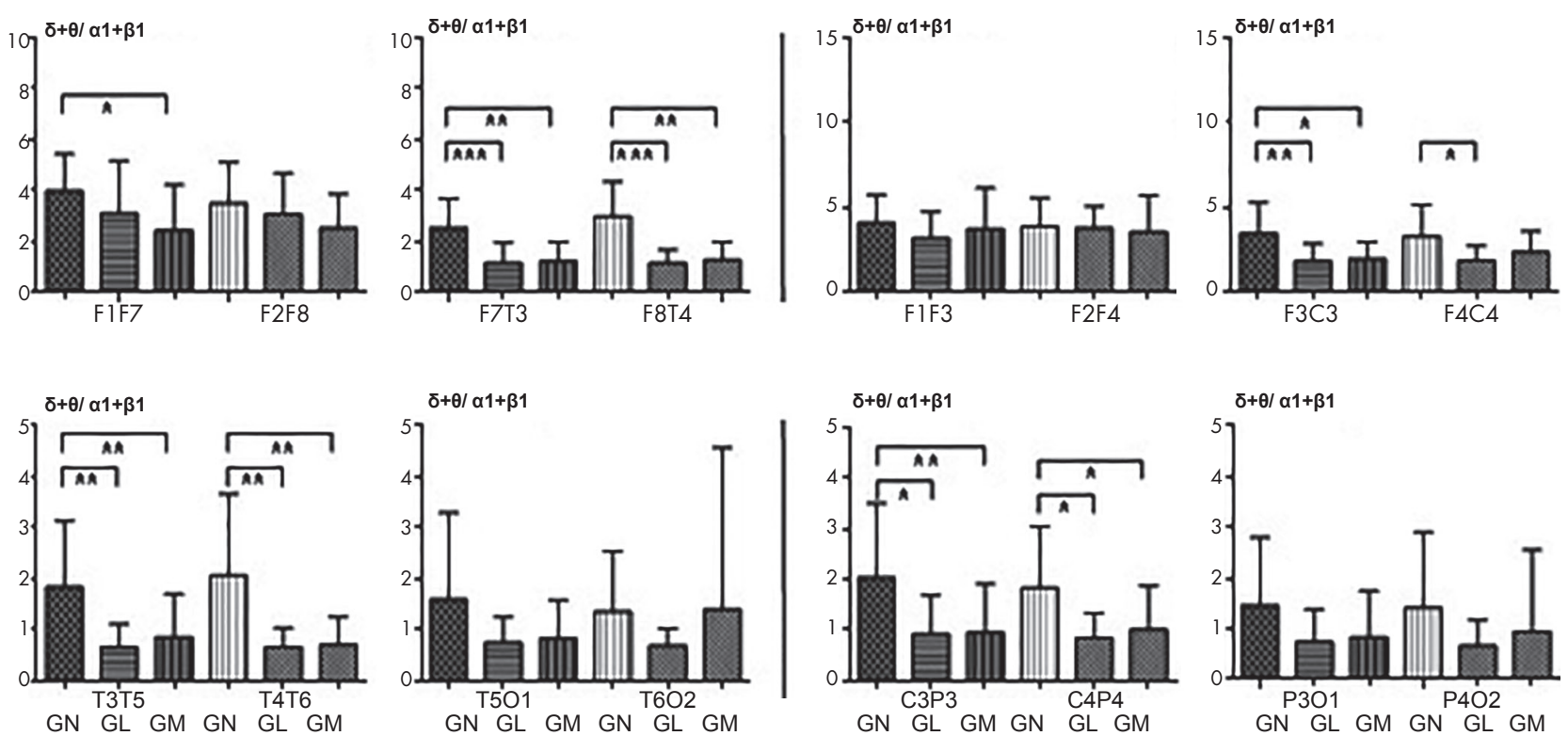

Figura 8. Las columnas representan los promedios, y la línea ascendente la desviación estándar de los índices $\precsim+\theta / \alpha 1+\beta 1$ en las diferentes derivaciones de cada grupo. Los asteriscos señalan el nivel de significancia entre las columnas indicadas.

encargan de integrar y coordinar la actividad de otros circuitos, quedando por último los que se relacionan con las funciones autonómicas y emocionales que parecen depender de las bajas frecuencias. ${ }^{18}$

Probablemente, la formación de circuitos está relacionada con la cantidad, intensidad y características de las activaciones sensoriales, con la integración de la información en relación con los estados de vigilia, con la motivación en diversos escenarios, así como con la especificidad de las respuestas conductuales correctas (aprendizaje y procesos cognitivos). ${ }^{21,35}$

La comunicación entre los circuitos se establece cuando, electrotónicamente, grupos de neuronas se despolarizan sincrónicamente, generando trenes de potenciales de acción que llevan una "clave" o se hiperpolarizan y se disminuyen dichos trenes, es decir inhibición por hiperpolarización. ${ }^{35-37}$

En esta hipótesis, se reúnen dos conceptos de las neurociencias: la sincronización de la actividad neuronal por oscilaciones espontáneas recurrentes y la descarga de los trenes de potenciales estocásticos. ${ }^{20,35,36}$ Los cuales, participan en los procesos cognitivos integrando dinámicamente los ensambles neuronales de circuitos cerebrales, principalmente en la conformación de redes que funcionan con ritmo $\alpha^{36-39}$ las cuales se forman y se integran en diferentes tiempos, generando importantes variaciones que se manifestaron en la mayor desviación estándar en los niños.

El incremento de $\theta$ con predominio en el HD en niños puede relacionarse con el inicio de los procesos de memorización al ocurrir en eventos específicos, ritmo que disminuye en el PA de los adultos, probablemente debido a que se transfiere a otras áreas cerebrales donde se almacena la información en diferentes ensambles glío-neuronales. ${ }^{11,20}$
Los resultados descritos permiten establecer, con mayores detalles, en tres diferentes grupos, la condición que recientemente se ha calificado de introspección en reposo con ojos cerrados, situación que se acepta como basal. De tal manera, que se pueden analizar los cambios del EEGc al abrir los ojos (extrospección) ${ }^{40}$ y continuar el análisis con paradigmas de atención y de aprendizaje. Los perfiles de PA de las cuatro bandas de frecuencia con su distribución topográfica, y los índices de $z$ y de $\theta$ sobre $\alpha$, se convierten en posibles indicadores funcionales.

El conocimiento de los mencionados indicadores EEGc permite identificar las variaciones morfo-funcionales en futuros EEG de niños, jóvenes y adultos, sometidos a procesos de diagnóstico de posibles alteraciones en su desarrollo o para localizar las lesiones adquiridas, ya sea por traumatismos cerebrales o por enfermedades cerebrovasculares. El presente trabajo tiene, no obstante, una importante limitación, representada por la magnitud de la muestra y la falta de una muestra de estudiantes de secundaria.

\section{Financiamiento}

Este trabajo quedó dentro del protocolo de financiamiento del CONACyT Salud 11 \# 161587.

\section{Conflicto de intereses}

Los autores declararon no tener conflicto de intereses.

\section{Agradecimientos}

A los doctores Blanca Flores Ávalos y Teodoro Flores por la revisión analógica de los registros. A Ingrid Mascher por la revisión del manuscrito. 


\section{REFERENCIAS}

1. Mountcastle VB. The columnar organization of the neocortex. Brain 1997;120:701-722.

2. Barry RJ, Clarke AR, Johnstone SJ, Magee CA et al. EEG differences between eyes-closed and eyes-open resting conditions. Clin Neurophysiol 2007;118:2765-2773.

3. Ben-Simon E, Podlipsky I, Arieli A, Zhdanov A et al. Never resting brain: simultaneous representation of two alpha related processes in humans. PloS One 2008;3:e3984.

4. Poskanzer KE, Yuste R. Astrocytic regulation of cortical UP states. PNAS 2011;108:18453-18458.

5. Van Dijk H, Schoffelen JM, Oostenveld R, Jensen O. Prestimulus oscillatory activity in the alpha band predicts visual discrimination ability. J Neurosci 2008;28:1816-1823.

6. Varela F, Lachaux JP, Rodriguez E, Martinerie J. The brainweb: phase synchronization and large-scale integration Nat Rev Neurosci 2001;2:229-239.

7. Sporns O. From complex networks to intelligent systems. En: Creating brain like intelligence. Sendhoff B, Körner E, Sporns O, Ritter H, Doya K (eds.). Berlin, Heidelberg: Springer Verlag; 2008.

8. Thatcher RW, Lubar JF. History of the scientific standards of QEEG normative databases. En: Budzinsky T, Budzinski H, Evans J, Abarbanel A (eds.). Introduction to QEEG and neurofeedback: Advanced theory and applications. San Diego, CA: Academic Press; 2008.

9. Başar E, Güntekin B. A short review of alpha activity in cognitive processes and in cognitive impairment. Int J Psychophysiol 2012;86:25-38.

10. Kopell N, Kramer MA, Malerba P, Whittington MA. Are different rhythms good for different functions? Front Hum Neurosci 2010;4:187. doi:10.3389/fnhum.2010.00187.

11. Tononi J. An information integration theory of consciousness. BMC Neurosci 2004;5:42-64.

12. Buzsáki G. Rhythms of the brain. Oxford: University Press; 2006.

13. Hensch TK. Critical period plasticity in local cortical circuits. Nat Rev Neurosci 2005;6:877-888.

14. Taylor AG, Goehler LE, Galper DI, Innes KE et al. Top-down and bottom-up mechanisms in mind-body medicine: development of an integrative framework for psychophysiological research. Explore (NY) 2010;6:29-41.

15. Engel AK, Fries $P$, Singer W. Dynamic predictions: oscillations and synchrony in top-down processing. Nat Rev Neurosci 2001;2:704-716.

16. Başar E. A review of alpha activity in integrative brain function: fundamental physiology, sensory coding, cognition and pathology. Int J Psychophysiol 2012;86:1-24.

17. Accolla EA, Kaplan PW, Maeder-Ingvar M, Jukopila S et al. Clinical correlates of frontal intermittent rhythmic delta activity (FIRDA). Clin Neurophysiol 2011;122:27-31.

18. Knyazev GG. EEG delta oscillations as a correlate of basic homeostatic and motivational processes. Neurosci Biobehav Rev 2012;36:677-695.

19. Sokolova LS, Machinskaia RI. Formation of the functional organization of the cerebral cortex at rest in young schoolchildren varying in the maturity of cerebral regulatory systems: I. Analysis of EEG spectral characteristics in the state of rest. Human Physiology 2006;32(5):499-508.

20. Catani M, Ffytche DH. The rises and falls of disconnection syndromes. Brain 2005;128:2224-2239.

21. Fingelkurts AA, Fingelkurts AA. Persistent operational synchrony within brain default-mode network and self-processing operations in healthy subjects. Brain Cogn 2011;75:79-90. doi: 10.1016/j.bandc.2010.11.015.

22. Palva S, Palva JM. New vistas for a-frequency band oscillations. 2007;30:150-158.

23. Steriade, M. Cellular substrates of brain rhythms. En: Niedermeyer E, Lopes da Silva FH (eds.). Electroencephalography: Basic principles, clinical applications, and related fields. Philadelphia: Williams \& Wilkins; Cuarta edición; 1998.

24. Wang XJ. Neurophysiological and computational principles of cortical rhythms in cognition. Physiol Rev 2010;90:1195-1268.

25. Buxhoeveden DP, Casanova MF. The minicolumn hypothesis in neuroscience. Brain 2001;125:935-951.

26. Lopes da Silva F. Principles of neural coding from EEG signals. En: Quiroga R, Panzeri S (eds.). Principles of neural coding. Boca Raton, FL: Taylor \& Francis Group: CRC Press; 2013.

27. Steriade M. Cellular substrates of brain rhythms. En: Niedermeyer E, Lopes da Silva F (eds.). Electroencephalography basic principles, clinical applications, and related fields. Philadelphia: Cuarta edición; Lippincott Williams \& Wilkins; 1998.

28. Llinás RR, Steriade M. Bursting of thalamic neurons and states of vigilance. J Neurophysiol 2006;95:3297-3308.

29. Jang JW, An S, Sun JJ, Reyes-Puerta V et al. Thalamic network oscillations synchronize ontogenetic columns in the newborn rat barrel cortex. Cereb Cortex 2013;23:1299-1316.

30. Rektor I, Kuba R, Brázdil M. Interictal and ictal EEG activity in the basal ganglia: an SEEG study in patients with temporal lobe epilepsy. Epilepsia 2002;43:253-262.

31. Rosanova M, Casali A, Bellina V, Resta F et al. Natural frequencies of human corticothalamic circuits. J Neurosci 2009;29:7679-7685; doi: 10.1523/JNEUROSCI.0445-09.2009.

32. Grossberg S. How does the cerebral cortex work? Learning, attention, and grouping by the laminar circuits of visual cortex. Spat Vis 1999;12:163-185.

33. Lopes da Silva F. Neural mechanisms underlying brain waves: from neural membranes to networks, Electroencephalogr Clin Neurophysiol 1991;79:81-93.

34. Schroeder CE, Lakatos P. Low-frequency neuronal oscillations as instruments of sensory selection. Trends Neurosci 2009;32:9-18; doi: 10.1016/j.tins.2008.09.012.

35. Machinskaia RI, Sokolova LS, Krupskaia EV. Formation of the functional organization of the cerebral cortex at Rest in young schoolchildren varying in the maturity of cerebral regulatory systems: II. Analysis of EEG a -Rhythm Coherence. Human Physiology 2007;33:129-138.

36. Fries P. A mechanism for cognitive dynamics: neuronal communication through neuronal coherence Trends Cogn Sci 2005;9:474-480.

37. Quian Quiroga R, Panzeri S. Principles of neural coding. CRC Press: Taylor 6 Francis Group; 2013.

38. Raichle ME. Two views of brain function. Trends Cog Sci 2010;14:180190.

39. Timofeev I, Bazhenov M, Seigneur J, Sejnowski T. Neuronal synchronization and thalamocortical rhythms in sleep, wake and epilepsy. En: Noebels JL, Avoli M, Rogawski MA et al. (eds.). Jasper's Basic mechanisms of the epilepsies. Bethesda, MD: NCBI; 2012.

40. Xu P, Huang R, Wang J, Van Dam NT et al. Different topological organization of human brain functional networks with eyes open versus eyes closed. NeuroImage 2014;90:246-255. 